\title{
SYNTHESIS AND BIOLOGICAL PROPERTIES OF SELECTED 2-ARYL-4(3H)-QUINAZOLINONES
}

\author{
Eung Seok Lee, Jong Keun Son, Young Hwa Na, Yurngdong Jahng* \\ College of Pharmacy, Yeungnam University, Kyongsan 712-749, Korea
}

\begin{abstract}
A series of 2-aryl-4(3H)quinazolinones were prepared as parent systems of rutaecarpine and luoitonin A and their biological properties (cytotoxicity and COX-2 inhibitory activity) were evaluated.
\end{abstract}

Attractiveness of alkaloids rutaecarpine (1) (1) and luotonin A (2) (2) stemmed from their promising cytotoxicities $\left(\mathrm{IC}_{50}\right.$ 's $=18.9$ and $6.6 \mu \mathrm{M}$, respectively, for human leukemia cancer cell line) (3) as well as a variety of intriguing biological properties including anti-inflammatory activity (4). We have recently involve in the synthesis and selective inhibitory activities of in 1 and 2 as well as their homologues on COX-2, in which the inhibitory activities are highly dependent on the length of the methylene bridge connecting $\mathrm{N} 3$ of $4(3 \mathrm{H})$-quinazolinone and $\mathrm{C} 2$ of either $1 \mathrm{H}$-indole or quinoline in 1 and 2 , respectively (5).<smiles>O=c1c2ccccc2nc2n1CCc1c-2[nH]c2ccccc12</smiles>

1<smiles>O=c1c2ccccc2nc2n1Cc1cc3ccccc3nc1-2</smiles>

2

Previous studies on the conformations of annulated aza-biaryls such as 3,3'-polymethylene-2,2'-bipyridines (6) and 3,2'polymethylene-2-phenylpyridines ( 7 ) revealed that the conformations of the unbridged parent compounds lay in between di- and tri-methylene bridged system. The relationship between the length of methylene-bridge and the dihedral angles of the 3,3'polymethylene-2-(1H-indol-2-yl)-4(3H)-quinazolinones are shown in Figure 1. We, thus, reason that the non-bridged parent compounds may have readily adoptable conformations for the corresponding receptor sites for the maximal biological activity.

Anti-inflammatory activity of well known indomethacin and our findings (5) on the biological properties of the rutaecarpine and luotonin $\mathrm{A}$ as well as their homologues on COX-2 and against selected human cancer cell lines prompted us to synthesize and evaluate biological properties of 2-aryl-4(3H)-quinazolinones (3) whose conformations would be in between di- and trimethylene bridged systems. 
Figure 1. Dihedral angle between 4(3H)-quinazolinone and $1 H$-indole ring<smiles></smiles>

\begin{tabular}{c|c}
\hline$X$ & Dihedral Angle, $\alpha^{\text {oa) }}$ \\
\hline$-\mathrm{CH}_{2}$ & 0 \\
$-\left(\mathrm{CH}_{2}\right)_{2^{-}}$ & $16\left(18,6.3^{\mathrm{b})}\right)$ \\
$-\left(\mathrm{CH}_{2}\right)_{3^{-}}$ & $52(35)$ \\
$\mathrm{H}, \mathrm{H}$ & $-(32)$ \\
\hline
\end{tabular}

Values are obtained from Dreiding Model and values in parenthesis are calculated from CS Chem3D Program. (8)

${ }^{b}$ Data was taken from X-ray crystal structure. (9)

\section{Synthesis}

The designed compounds 3 were prepared by employing previously reported method such as ammonolysis of $4 H-3,1-$ benzoxazolin-4-ones. (10) Although early studies claimed that the ammonolysis of 4H-3,1-benzoxazolin-4-ones with an aromatic substituent at $\mathrm{C} 2$ position required drastic reaction condition such as heating $240-250{ }^{\circ} \mathrm{C}(11)$ or with anhydrous $\mathrm{ZnCl}$,

(12) the reactions of 6 with $\mathrm{NH}_{4} \mathrm{OH}$ in $\mathrm{EtOH}$ under reflux afforded the desired product in good yield.

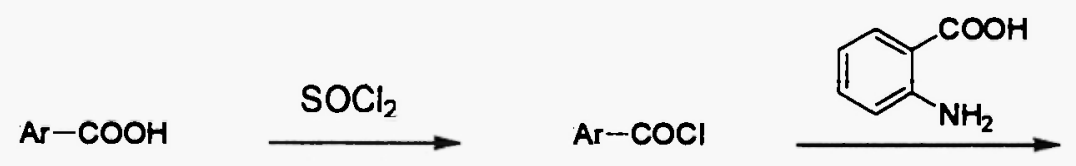

3

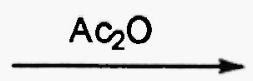<smiles>O=c1oc([Al])nc2ccccc12</smiles>

6<smiles>O=c1c2cc3ccccc3n2c(=O)c2cc3ccccc3n12</smiles>

8
4

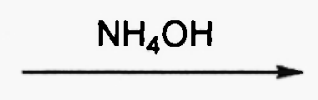<smiles>O=C([Al])Nc1ccccc1C(=O)O</smiles>

5
$7 \mathrm{a} \mathrm{Ar}=\mathrm{Ph}$

$\mathrm{b} \mathrm{Ar}=\widehat{N}_{\mathrm{N}^{\prime}}$

c Ar $=$

$\mathrm{dAr}=$ 
It is worthy to noting that the reaction of $1 \mathrm{H}$-indole-2-carboxylic acid in equimolar $\mathrm{SOCl}_{2}$ in dry $\mathrm{CHCl}_{3}$ has afforded $\mathrm{IH}$-indole2-carbonyl chloride in quantitative yield, although it has been claimed to afford bisindolodioxopiperazine (8). (13) Unlike dioxopiperazine 8, acid chloride $4 \mathrm{c}$ was very soluble in $\mathrm{CHCl}_{3}$ and shown a well-resolved proton resonances required for the structure. A resonance for $\mathrm{H} 3$ appeared at $\delta 7.55$ as an one-proton doublet $\left(J_{1.3}=2.1 \mathrm{~Hz}\right.$ ), which is downfiled-shifted by 0.16 $\mathrm{ppm}$ due to the stronger electron withdrawing effect of $\mathrm{COCl}$ at $\mathrm{C} 2$ compared to the parent carboxylic acid, confirmed such a conversion. (14) In addition, a broad $\mathrm{D}_{2} \mathrm{O}$-exchangeable singlet at $\delta 8.94$ also supported the presence of $\mathrm{N}-\mathrm{H}$. We were not able to either isolate or identify any trace of 8 . The bisindolodioxopiperazine (8), however, could be prepared by running the reaction in the presence of pyridine or triethylamine, which might uptake $\mathrm{HCl}$ formed, and thus catalyze formation of bisamide (8).

\section{Biological Properties}

COX Inhibitory Activity: Inhibitory activities of the compounds 7 on cyclooxygenase-1 and 2 (COX-1 and 2) were evaluated as compared to indomethacin and selective COX-2 inhibitor NS-398 by employing previously described method, (5) and summarized in Table 1 . The inhibitory activities on COX-1 and COX-2 were significantly increased in $7 \mathrm{c}$ and $7 \mathrm{~d}$ with COX-2 selectivity of 10.7 and 4.7 , respectively. Selectivity of $7 \mathrm{c}$ on $\mathrm{COX}-2$ is somewhat surprising compared to previous result of a loss of selectivity on triemthylene-bridged homologue of rutaecarpine. (5)

Cytotoxicity: Cytotoxicites of compounds 7 against human leukemia cancer cell line (CCRF-CEM) were evaluated by employing previously described method (15) and summarized in Table 1. Cytotoxicities of $7 c, d$ on CCRF-CEM are approximately 2- and 0.5 -fold increase compared to the parent rutaecarpine and luotonin $\mathrm{A}$, respectively, while 7a,b did not show any significant cytotoxicities up to $50 \mu \mathrm{M}$.

Table 1. Inhibitory Activities of Compound 7 on COX-1 and COX-2 and Human Cancer Cell Line

\begin{tabular}{|c|c|c|c|c|}
\hline \multirow{2}{*}{ Compounds } & \multicolumn{2}{|c|}{$\mathrm{IC}_{50}(\mu \mathrm{M})$} & \multirow{2}{*}{$\begin{array}{c}\text { Selectivity } \\
\text { nnnny }\end{array}$} & $\mathrm{IC}_{50}(\mu \mathrm{M})$ \\
\cline { 2 - 3 } \cline { 5 - 5 } & $\mathrm{COX}-1$ & $\mathrm{COX}-2$ & (COX-1/COX-2) & CCRF-CEM \\
\hline $7 \mathrm{a}$ & $>100$ & $>100$ & - & $>50$ \\
\hline $7 \mathrm{~b}$ & $>100$ & $>100$ & - & $>50$ \\
\hline $7 \mathrm{c}$ & 78.3 & 7.3 & 10.7 & 9.3 \\
\hline $7 d$ & 67.5 & 14.5 & 4.7 & 12.3 \\
\hline Indomethacin & 0.016 & 0.009 & 1.9 & \\
\hline NS-398 & 1.67 & $<0.002$ & $>8,300$ & \\
\hline
\end{tabular}

In conclusion, 2-(1H-indol-2-yl)-4(3H)-quinazolinone and 2-(quinol-2-yl)-4(3H)-quinazolinone were shown significant inhibitory activities on COX-2 and on cell growth of CCRF-CEM enough to be a possible lead for the future study as an antiinflammatory and/or antitumor agent. 


\section{Experimental}

Melting points were determined using a Fischer-Jones melting points apparatus and are not corrected. IR spectra were obtained using a Perkin-Elmer 1330 spectrophotometer. NMR spectra were obtained using a Bruker-250 spectrometer $250 \mathrm{MHz}$ or 400 $\mathrm{MHz}$ for ${ }^{1} \mathrm{H}$ NMR and $62.5 \mathrm{MHz}$ or $100 \mathrm{MHz}$ for ${ }^{13} \mathrm{C}$ NMR and are reported as parts per million (ppm) from the internal standard tetramethylsilane (TMS). The compounds $7 \mathrm{a}(16)$ and $7 \mathrm{~b}$ (17) were previously reported in the literature. Chemicals and solvents were commercial reagent grade and used without further purification. Elemental analyses were taken on a HewlettPackard Model 185B elemental analyzer.

\section{IH-Indole-2-carbonyl Chloride (4c)}

To a solution of indole-2-carboxylic acid $(3.64 \mathrm{~g}, 0.023 \mathrm{~mol})$ in $50 \mathrm{~mL}$ of $\mathrm{CHCl}_{3}$ at room temperature was added $\mathrm{SOCl}_{2}(5.51$ $\mathrm{mL}, 0.23 \mathrm{~mol}$ ). Resulting mixture was stirred for $8-12 \mathrm{~h}$ at room temperature and evaporated in vacuo to give $3.37 \mathrm{~g}(88 \%)$ of pale yellow solid. The obtained solid was used for the next step without any further purification. Unreported spectral data are as follows: IR (KBr) $\cup 3050,1750,1610,1475,1260,1245,1220,1180,981 \mathrm{~cm}^{1}$. 'H NMR (400 MHz, CDCl $) \delta 8.94$ (6r s, NH), 7.74 (dd, $J=8.9,0.8 \mathrm{~Hz}, \mathrm{H} 7$ ), 7.55 (d, $J=2.1 \mathrm{~Hz}, \mathrm{H} 3$ ), 7.45 (dd, $J=8.4,1.0 \mathrm{~Hz}, \mathrm{H} 4$ ), 7.43 (ddd, $J=8.4,7.8,0.8 \mathrm{~Hz}, \mathrm{H5}$ ), 7.23 (ddd, $J=8.4,8.0,1.0 \mathrm{~Hz}, \mathrm{H} 6$ ).

\section{Quinoline-2-carbonyl Chloride (4d)}

The same procedure described for $4 \mathrm{c}$ was employed with quinoline-2-carboxylic acid $(5.00 \mathrm{~g}, 0.029 \mathrm{~mol})$ to give $4.42 \mathrm{~g}(85 \%)$ of pale yellow needles (ether): $\mathrm{mp} 96-97^{\circ} \mathrm{C}$ [lit. (18) $\mathrm{mp} 96-97^{\circ} \mathrm{C}$ ]. Unreported spectral data are as follows: $\mathrm{IR}(\mathrm{KBr}) \cup 3260,2965$, $2890,1640,1488,1362,1090 \mathrm{~cm}^{-1}$. 'H NMR (400 MHz, CDCl 3 ) $\delta 8.35$ (d, $\left.J=8.8 \mathrm{~Hz}, \mathrm{H4}\right), 8.34$ (d, $\left.J=8.4 \mathrm{~Hz}, \mathrm{H8}\right), 8.13$ (d, $J=$ $8.8 \mathrm{~Hz}, \mathrm{H3}$ ), 7.92 (d, $J=8.0 \mathrm{~Hz}, \mathrm{H} 5$ ), 7.85 (td, $J=7.6,0.8 \mathrm{~Hz}, \mathrm{H} 7$ ), 7.73 (ddd, $J=7.6,1.0 \mathrm{~Hz}, \mathrm{H} 6$ ).

\section{$N$-(1H-Indole-2-carboyl)anthranilic acid (5c)}

To the acid chloride $4 \mathrm{c}(0.023 \mathrm{~mol})$ in $30 \mathrm{~mL}$ of $\mathrm{CHCl}_{3}$ was added anthranilic acid $(3.09 \mathrm{~g}, 0.023 \mathrm{~mol})$ in pyridine $(8 \mathrm{~mL})$ and $\mathrm{CHCl}_{3}(30 \mathrm{~mL})$. The reaction mixture was stirred for $8 \mathrm{~h}$ and refluxed for $\mathrm{lh}$. The pale yellow precipitate formed was collected and washed with $\mathrm{CHCl}_{3}$ to give $6.25 \mathrm{~g}(97 \%)$ as a pale green needles (EtOAc): $\mathrm{mp}>260{ }^{\circ} \mathrm{C}$. IR (KBr) $\cup 3305,3049,1745,1741$, 1630, 1605, 1474, 1264, 1236, 1220, 1180, 973, $764 \mathrm{~cm}^{-1}$. 'H NMR (DMSO-d6, $400 \mathrm{MHz}$ ) $\delta 12.28$ (s, 1H), 11.96 (s, 1H), 8.72 (d, $J=7.6 \mathrm{~Hz}, 1 \mathrm{H}), 8.07$ (d, $J=8.0 \mathrm{~Hz}, 1 \mathrm{H}), 7.71(\mathrm{~d}, J=8.0 \mathrm{~Hz}, 1 \mathrm{H}), 7.67$ (d, $J=8.4 \mathrm{~Hz}, 1 \mathrm{H}), 7.49(\mathrm{~d}, J=8.4 \mathrm{~Hz}, 1 \mathrm{H}), 7.25(\mathrm{td}, J$ $=8.0,0.8 \mathrm{~Hz}, 1 \mathrm{H}$ ), $7.19(\mathrm{t}, J=7.6 \mathrm{~Hz}, 1 \mathrm{H}), 7.13\left(\mathrm{~s}, 1 \mathrm{H}, \mathrm{H} 3^{\prime}\right), 7.09$ (td, $J=7.6,0.8 \mathrm{~Hz}, 1 \mathrm{H}$ ). Anal. Calcd for $\mathrm{C}_{16} \mathrm{H}_{12} \mathrm{~N}_{2} \mathrm{O}_{3} 1 / 2 \mathrm{H}_{2} \mathrm{O}$ : C, 66.43; H, 4.53; N, 9.68. Found: C, 66.36; H, 4.52; N, 9.70 .

\section{N-(Quinoline-2-carbonyl)anthranilic acid (5d)}

The same procedure described for $5 \mathrm{C}$ was employed with $4 \mathrm{~d}(0.023 \mathrm{~mol})$ to give $6.18 \mathrm{~g}(92 \%)$ pale yellow needles (EtOAc): $\mathrm{mp}$ 241-242 ${ }^{\circ} \mathrm{C}$. IR (KBr) $\cup 3306,3050,1745,1740,1631,1604,1473,1265,1235,1221,972 \mathrm{~cm}^{1} .{ }^{\prime} \mathrm{H}$ NMR (DMSO-d $\left.d_{5}\right), 400$ MHz) $\delta 13.43(\mathrm{~s}, 1 \mathrm{H}), 8.92(\mathrm{~d}, J=8.4 \mathrm{~Hz}, 1 \mathrm{H}), 8.65(\mathrm{~d}, J=8.4 \mathrm{~Hz}, 1 \mathrm{H}), 8.29$ (d, $J=8.4 \mathrm{~Hz}, 1 \mathrm{H}), 8.13(\mathrm{~d}, J=8.4 \mathrm{~Hz}, 2 \mathrm{H}), 8.09$ (dd, $J=8.4,1.4 \mathrm{~Hz}, 1 \mathrm{H}$ ), 7.92 (td, $J=8.4,1.6 \mathrm{~Hz}, 1 \mathrm{H}$ ), 7.77 (td, $J=8.4,1.2 \mathrm{~Hz}, 1 \mathrm{H}$ ), 7.70 (td, $J=8.4,1.5 \mathrm{~Hz}, 1 \mathrm{H}$ ), 7.67 (td, $J=$ 
8.4, $1.6 \mathrm{~Hz}, 1 \mathrm{H}$ ), 7.24 (td, $J=8.4,1.2 \mathrm{~Hz}, 1 \mathrm{H}$ ). ${ }^{13} \mathrm{C}$ NMR (DMSO- $\left.\tau_{6}, 100 \mathrm{MHz}\right) \delta 169.4,163.1,149.9,146.1,140.7,138.7$, 134.5, 131.8, 131.1, 129.5, 129.4, 128.8, 128.5, 123.4, 120.0, 118.9, 117.3. Anal. Calcd for $\mathrm{C}_{17} \mathrm{H}_{12} \mathrm{~N}_{2} \mathrm{O}_{3} \cdot \mathrm{H}_{2} \mathrm{O}: \mathrm{C}, 66.43 ; \mathrm{H}, 4.53$; $\mathrm{N}, 9.68$. Found: C, 66.36; H, 4.52; N, 9.70.

\section{2-(1H-Indol-2-yl)-4H-3,1-benzorazolin-4-one (6c)}

A suspension of $5 \mathrm{c}(1.40 \mathrm{~g}, 0.005 \mathrm{~mol})$ in $\mathrm{Ac}_{2} \mathrm{O}(10 \mathrm{~mL})$ was refluxed for $2 \mathrm{~h}$. Evaporation of the solvent in vacuo gave pale yellow solid, which was suspended in water and stirred for $30 \mathrm{~min}$. The precipitate formed was collected and washed with water, followed by EtOH to give $1.26 \mathrm{~g}$ (96\%) of pale yellow needles: $\mathrm{mp} 210-212{ }^{\circ} \mathrm{C}$ [lit. (19) $\mathrm{mp} 198-200{ }^{\circ} \mathrm{C}$ ]. Unreported spectral data are as follows: IR (KBr) $\cup 3296,1661,1607,1540,1450,1412,1315,1262 \mathrm{~cm}^{-1}$. ${ }^{1} \mathrm{H}$ NMR (DMSO-do, $\left.400 \mathrm{MHz}\right) \delta 11.32$ (s, $1 \mathrm{H}, \mathrm{N}-\mathrm{H}), 8.10$ (d, $J=7.9 \mathrm{~Hz}, 1 \mathrm{H}), 7.76(\mathrm{~d}, J=7.9 \mathrm{~Hz}, 1 \mathrm{H}), 7.60-7.55(\mathrm{~m}, 2 \mathrm{H}), 7.47$ (d, $J=8.4 \mathrm{~Hz}, 1 \mathrm{H}), 7.43$ (t, $J=8.0 \mathrm{~Hz}$, $1 \mathrm{H}), 7.29$ (s, $1 \mathrm{H}, \mathrm{H} 3), 7.19$ (t, $J=7.2 \mathrm{~Hz}, 1 \mathrm{H}), 7.02$ (td, $J=7.6,0.8 \mathrm{~Hz}, 1 \mathrm{H}$ ). ${ }^{13} \mathrm{C}$ NMR NMR (DMSO-d, $\left.100 \mathrm{MHz}\right) \delta 157.9$, $151.5,146.1,137.5,135.7,127.6,126.9,126.8,126.6,125.6,124.0,120.9,119.5,115.8,111.6,106.9$.

\section{2-(Quinol-2-yl)-4H-3,1-benzorazolin-4-one (6d)}

The same procedure described above for $6 \mathrm{C}$ was employed with $5 \mathrm{~d}(1.46 \mathrm{~g}, 0.005 \mathrm{~mol})$ to give $1.26 \mathrm{~g}$ (92\%) of pale pink needles (EtOAc): $\mathrm{mp} 182-183{ }^{\circ} \mathrm{C}$. IR (KBr) $\cup 3297,1662,1610,1540,1450,1412,1315,1261 \mathrm{~cm}^{-1} .^{1} \mathrm{H}$ NMR (DMSO-d, $400 \mathrm{MHz}$ ) $\delta 8.60(\mathrm{~d}, J=8.9 \mathrm{~Hz}, 1 \mathrm{H}), 8.46(\mathrm{~d}, J=8.4 \mathrm{~Hz}, \mathrm{HH}), 8.24(\mathrm{~d}, J=8.0 \mathrm{~Hz}, \mathrm{H}), 8.23(\mathrm{td}, J=7.6,0.8 \mathrm{~Hz}, 1 \mathrm{H}), 8.11(\mathrm{~d}, J=8.4 \mathrm{~Hz}$, $1 \mathrm{H}$ ), 8.01 (td, $J=8.0,1.4 \mathrm{~Hz}, 1 \mathrm{H}$ ), 7.90 (ddd, $J=8.0,7.6,0.8 \mathrm{~Hz}, 1 \mathrm{H}$ ), 7.84 (d, $J=8.0 \mathrm{~Hz}, 1 \mathrm{H}$ ), 7.76 (td, $J=8.0,0.8 \mathrm{~Hz}, 1 \mathrm{H}$ ), $7.71\left(\mathrm{td}, J=7.6,1.0 \mathrm{~Hz}, 1 \mathrm{H}\right.$ ). ${ }^{13} \mathrm{C}$ NMR NMR (DMSO-do, $\left.100 \mathrm{MHz}\right) \delta 159.0,155.3,148.0,147.0,145.9,137.5,136.9,130.7$, 129.7, 129.5, 128.6, 128.6, 128.1, 128.1, 127.3, 120.3, 117.6. Anal. Calcd for $\mathrm{C}_{17} \mathrm{H}_{12} \mathrm{~N}_{2} \mathrm{O}_{2}: \mathrm{C}, 74.45 ; \mathrm{H}, 3.67 ; \mathrm{N}, 10.21$. Found: $\mathrm{C}$, $74.25 ; \mathrm{H}, 3.74 ; \mathrm{N}, 10.31$.

\section{2-(1H-Indol-2-yl)-4(3H)-quinazolinone (7c)}

A solution of $6 \mathrm{c}(1.32 \mathrm{~g}, 0.005 \mathrm{~mol})$ and $35 \% \mathrm{NH}_{4} \mathrm{OH}(35 \mathrm{~mL})$ in $\mathrm{EtOH}(200 \mathrm{~mL})$ was refluxed for $6 \mathrm{~h}$ with addition of additional $35 \% \mathrm{NH}_{4} \mathrm{OH}(35 \mathrm{~mL})$ every $2 \mathrm{~h}$. The creamy precipitate formed was collected and washed with water to give $2.09 \mathrm{~g}$ (80\%) of solid which was purified by sublimation. mp 319-320 ${ }^{\circ} \mathrm{C}$ [lit. (19) $\mathrm{mp} 318-320^{\circ} \mathrm{C}$ ]. ${ }^{1} \mathrm{H}$ NMR (DMSO-d6 $400 \mathrm{MHz}$ ) $\delta 12.62$ (s, Nl'-H), 11.80 (s, N3-H), 8.17 (dd, $J=7.9,1.2 \mathrm{~Hz}, \mathrm{H} 5$ ), 7.84 (td, $J=7.9,0.8 \mathrm{~Hz}, \mathrm{H} 7$ ), 7.74 (dd, $J=8.0,1.0 \mathrm{~Hz}, \mathrm{H} 8$ ), 7.68 (s, H3'), 7.65 (dd, $J=8.0,0.8 \mathrm{~Hz}, \mathrm{H}^{\prime}$ ), 7.54 (dd, $\left.J=8.0,1.2 \mathrm{~Hz}, \mathrm{H7} 7^{\prime}\right), 7.50$ (t, $J=8.0 \mathrm{~Hz}, \mathrm{H6}$ ), 7.23 (t, $J=7.6 \mathrm{~Hz}, \mathrm{H6} 6^{\prime}$ ), 7.06 (t. $J=7.6 \mathrm{~Hz}, \mathrm{H} 5$ ). ${ }^{13} \mathrm{C}$ NMR NMR (DMSO- $d_{6,} 100 \mathrm{MHz}$ ) $\delta 161.8,148.8,146.6,137.7,134.7,130.0,127.5,126.9,126.3$, $126.1,124.1,121.5,121.2,120.0,112.4,105.0$.

\section{2-(Quinol-2-yl)-4(3H)-quinazolinone (7d)}

The same procedure described above for $7 \mathrm{c}$ was employed with $6 \mathrm{~d}(2.74 \mathrm{~g}, 0.01 \mathrm{~mol})$ to give $2.05 \mathrm{~g}$ (75\%) of fine white needles $\left(\mathrm{CHCl}_{3}\right): \mathrm{mp} \mathrm{267-268}{ }^{\circ} \mathrm{C}$. IR $(\mathrm{KBr}) \cup 3376,3066,1649,1603,1581,1524,1504,1451,1404,1297,1265,770,752 \mathrm{~cm}^{-1} .{ }^{1} \mathrm{H}$ NMR (DMSO-d $\left.d_{6,} 400 \mathrm{MHz}\right) \delta 8.80$ (d, $\left.J=8.4 \mathrm{~Hz}, 1 \mathrm{H}\right), 8.42(\mathrm{~d}, J=8.4 \mathrm{~Hz}, 1 \mathrm{H}), 8.25(\mathrm{~d}, J=8.4 \mathrm{~Hz}, 1 \mathrm{H}), 8.13(\mathrm{~d}, J=8.4 \mathrm{~Hz}$, 1H), 7.94 (d, $J=8.4 \mathrm{~Hz}, 1 \mathrm{H}$ ), 7.80 (dd, $J=8.4 .1 .4 \mathrm{~Hz}, 1 \mathrm{H}$ ), 7.78 (ddd, $J=8.4,8.0,1.0 \mathrm{~Hz}, 1 \mathrm{H}$ ), 7.63 (ddd, $J=8.4,8.0,1.0 \mathrm{~Hz}$, 
$1 \mathrm{H}$ ), 7.48 (ddd, $J=8.4,8.0,1.0 \mathrm{~Hz}, 1 \mathrm{H}$ ), 7.10 (ddd, $J=8.4,8.0,1.0 \mathrm{~Hz}, 1 \mathrm{H}$ ). ${ }^{13} \mathrm{C}$ NMR (DMSO-d, $100 \mathrm{MHz}$ ) $\delta 170.5,162.6$, $149.7,145.8,138.7,137.3,131.5,129.8,129.3,128.7,128.3,127.7,127.4,122.3,120.9,120.1,118.3$. Anal. Calcd for $\mathrm{C}_{17} \mathrm{H}_{11} \mathrm{~N}_{3} \mathrm{O} \mathrm{H}_{2} \mathrm{O}: \mathrm{C}, 70.09 ; \mathrm{H}, 4.50 ; \mathrm{N}, 14.43$. Found. $\mathrm{C}, 70.16 ; \mathrm{H}, 4.52 ; \mathrm{N}, 14.39$.

Acknowledgement: Support by Korean Research Foundation Grant (KRF-2002-005-E00019) is gratefully acknowledged.

\section{References}

(1) a) Y. Ashima and K. Kashiwali, J. Pharm. Soc. Jpn 35, 1293 (1915). b) E. S. Lee, J. G. Park, and Y. Jahng, Tetrahedron Lett. 44, 1883 (2003), and references therein.

(2) a) Z.-Z. Ma, Y. Hano, T. Nomura, and Y.-J. Chen, Heterocycles 46, 541 (1997). b) A. Cagir, S. H. Jones, R Gao, B. M. Eisenhauer, and S. M. Hecht, J. Am. Chem. Soc. 125, 13628 (2003), and references therein.

(3) a) L.-M. Yang, C.-F. Chen, and K.-H Lee, Bioorg. Med. Chem. Lett. 5,465 (1995). b) Z-Z. Ma, Y. Hano, T. Nomura, and Y.J. Chen, Heterocycles 55, 1555 (2001).

(4) a) T. C. Moon, M. Murakami, I. Kudo, K. H. Son, H. P. Kim, S. S. Kang, and H. W. Chang, Inflamm Res. 48, 621 (1999). b) P.-G. Xiao, G.-L. Quo, H.-L Wang, L.-S. Lui, Y.-L. Zheng, Z.-J. Jia, and Z.-B. Deng, Chinese J. Pharmacol. Toxicol. 232 (1988). c) Y. Jahng, Unpublished results.

(5) a) H. W. Chang, S. I. Kim, H. Jung, and Y. Jahng, Heterocycles 60, 1359 (2003). b) Y. Jahng, Unpublished results

(6) R. P. Thummel, F. Lefoulon, and R. Mahadevan, J. Org. Chem 50, 3824 (1985).

(7) R. P. Thummel and Y. Jahng, J. Org. Chem 52, 71 (1987).

(8) Available from CambridgeSoft Corporataion, Cambridge, MA 02140-2317, USA.

(9) I. Fujii, Y. Kobayashi, and N. Hirayama, Z. Kristallogr. 215, 762 (2000).

(10) C. A. Fetscher and M. T. Bogert, J. Org. Chem. 4, 71 (1939).

(11) a) W. L. F. Armarego, Part I Quinazolines in Fused Pyrimidines ed. by D. J. Brown, Interscience Publishers, New York, 1967, and references therein b) M. Claesen and H. Vanderhaeghe, Bull. Soc. Chim. Belges. 68, 220 (1959). c) S. S. Joshi and I. R. Gambhir, J. Org. Chem. 26, 3714 (1961).

(12) D. T. Zentmeyer and E. C. Wagner, J. Org. Chem. 14, 967 (1949).

(13) A.-R. Lee, W.-H. Huang, and J. -Y. Cheng, Chin. Pharm. J. 46, 307 (1994).

(14) G. Cignarella, D. Barlocco, P. Vianello, S. Villa, G. A. Pinna, P. Fadda, W. Fratta, L. Toma, and S. Gessi, II Pharmaco 53, 667 (1998). They reported $1 H$-indole-2-carbonyl chloride as an intermediate without any physical and spectral data.

(15) M. R. Boyd, Princ, Pract. Oncol. 3, 1 (1989)

(16) M. T. Bogert, R. A. Gortner, and C. G. Amend, J. Am. Chem. Soc. 33, 949 (1911).

(17) L. Berezowski and W. Dymek, Acta Poloniae Pharm 27, 11 (1970).

(18) J. W. David, JR. J. Org. Chem. 24, 1691 (1959).

(19) J. Bergman and N. Eklund, Chemica Scripta 19, 193 (1982).

Received on February 4, 2004. 